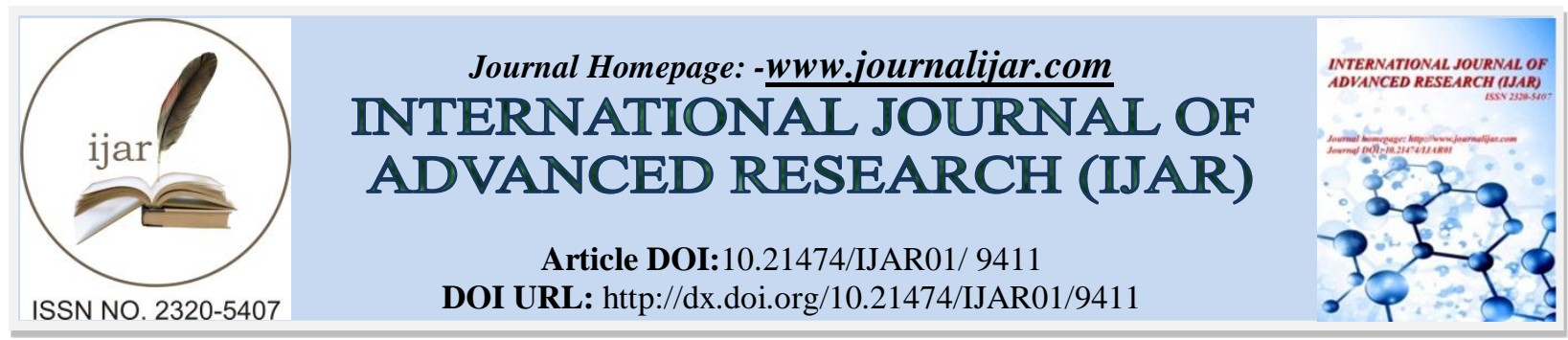

RESEARCH ARTICLE

\title{
CASE STUDY ON BILATERAL STASIS DERMATITIS WITH CELLULITIS - A PHARMACIST OUTLOOK.
}

\author{
S. Kavitha, S. Sabishruthi and B. Jagan Nathan. \\ Department of Pharmacy Practice, Jaya College of Paramedical Sciences, College of Pharmacy, Thiruninravur, \\ Chennai- 602024.
}

\section{Manuscript Info}

Manuscript History

Received: 20 May 2019

Final Accepted: 22 June 2019

Published: July 2019

Key words:-

Stasis dermatitis, Cellulitis, Therapy,

Counselling, Quality of life.

\section{Abstract}

Background: Stasis dermatitis is a common clinical manifestation present with varicose vein, skin changes such as dermatitis hyperpigmentation, lipodermatosclerosis and chronic leg ulcers. Cellulitis occurs when the bacteria enter the skin and spread which results in redness, painful swelling or warmth.

Case Presentation: A 52 years old male patient was admitted in a Tertiary care hospital at Thiruvallur with the chief complaints of swelling and open sore for two weeks and itching for about 10 days and redness since 4 days. Later he developed skin lesions and cracking skin in both the legs with exudation. The patient was diagnosed to have stasis dermatitis with cellulitis. The patient was treated with topical applications and antibiotics to improve the clinical condition. Conclusion: The patient was treated with appropriate drug therapy with proper counselling sessions regarding disease, drugs and lifestyle modifications. The pharmacist plays an important role in analyzing the case study in order to provide excellence in clinical outcomes and to improve the patient's quality of life.

Copy Right, IJAR, 2019,. All rights reserved.

\section{Introduction:-}

Stasis dermatitis is a cutaneous sign of venous insufficiency. It usually presents with erythema, scale, pruritus, erosions, exudate and crust. It is mostly common in lower extremities. It may be bilateral or unilateral edema is often present as well as various veins and hemosiderin ${ }^{[1-5]}$. It occurs in valves which is present in leg vein. It is commonly caused by staphylococci aureus or herpes simplex virus and also caused by certain drugs like amlodipine and corticosteroids ${ }^{[4-8]}$. The pathogenesis of venous disease is due to swelling of incompetent veins that cause edema and inflammation. It tends to causes cellulitis, contact dermatitis and pigmented purpuric dermatoses ${ }^{[11]}$. It commonly affects people belong to low socio-economic status which compels the people to change their job out of compulsion which further affects the quality of life of the individual. It is a common condition affects adolescents to elderly. The etiology of the disease is not completely understood ${ }^{[3]}$.

\section{Case presentation:}

A 52 years old male patient was admitted in the Tertiary care hospital at Thiruvallur with the chief complaints of swelling and open sore for two weeks and itching for about 10 days and redness since 4 days. Later he developed

Corresponding Author:-S. Kavitha. 
skin lesions and cracking skin in both the legs with exudation [Fig (a), (b) \& (c)]. Vitals of the patient were seems to be normal.

Fig (a), (b) \& (c):- represent the condition of the patient

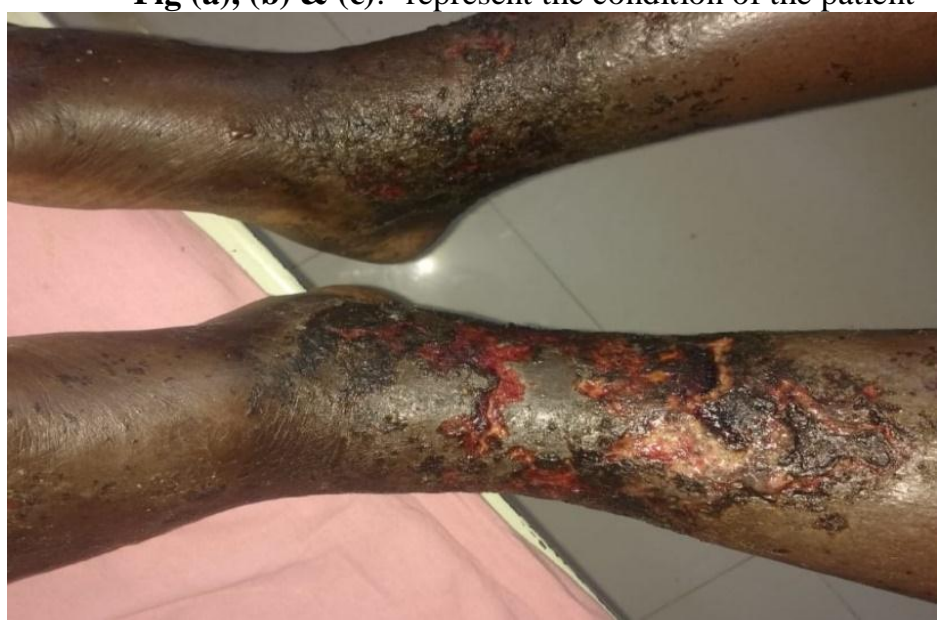

The figure shows patients with skin lesions

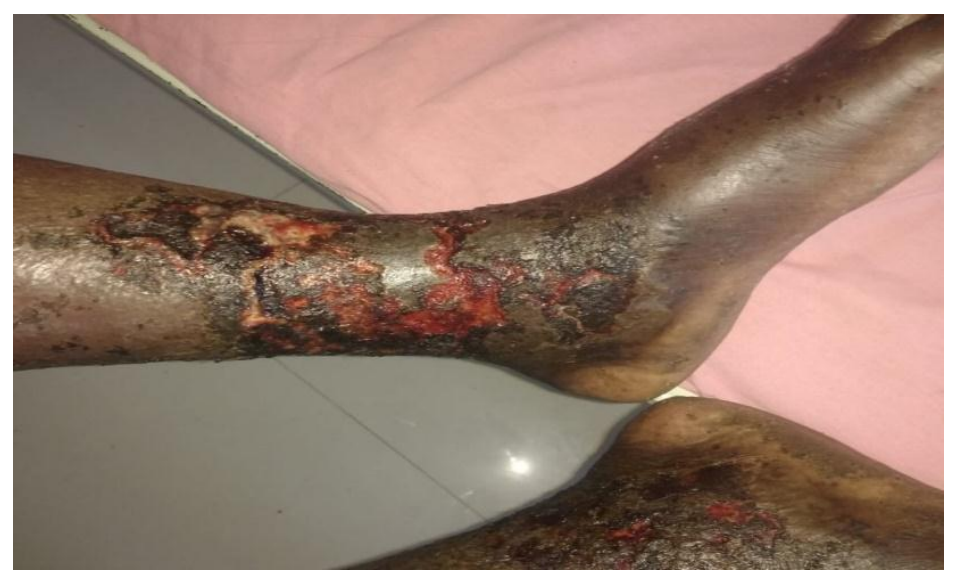

The figure represents the condition with cracking of skin



The figure represents condition with swelling and open sore in both legs

On examination, the patient was conscious, oriented, afebrile and non-anemic at the time of admission. The laboratorial investigation reveals that the patient has increased WBC count to $11,730 \mu 1$ and increased blood urea 
nitrogen to $29 \mathrm{mg}$. The abdomen evaluation revealed that per abdomen was soft, and other observed parameters are normal including systemic examination.

The patient had no similar family history. He is a non-alcoholic and non-smoker. The patient was a known case of hypertension. He belongs to low socio economic status and has normal personals habits and behavior. The patient was taking Tablet Amlodipine 5mg for the past 6 months. He takes mixed diet and temporary insomniac ( due to pain in the infected area).

The patient was treated initially with Inj. Ceftriaxone, Inj. Diclofenac, Inj. Amoxicillin, T. Vitamin B complex and Mupirocin $2 \%$ ointment as a topical application. The patient was kept under observation to improve the clinical condition.

The patient was reviewed weekly. Open sores and cracks were healed significantly in legs. After drug therapy and strict counselling were followed for 4 weeks; the patient condition was seemed to be improved compared to past. Here figure (d) represents the condition after the treatment and counselling sessions.

Fig d:-shows the condition after treatment

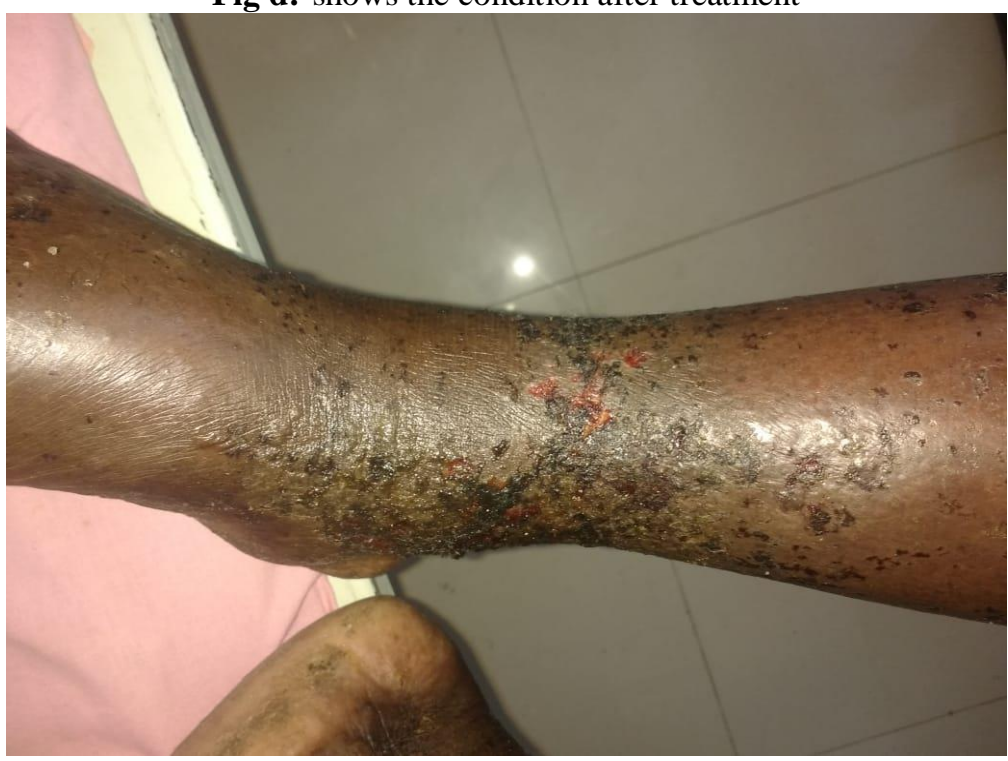

\section{Discussion:-}

Stasis dermatitis is a common condition that occurs secondary to venous hypertension. It is usually present with eczematous lesion around the ankle and lower legs in the common cutaneous sites which gets affected by chronic ambulatory venous hypertension. Chronic venous disease is common with manifestations that include varicose veins, skin changes such as dermatitis hyperpigmentation, lipodermatosclerosis and chronic leg ulcers ${ }^{[10]}$. Venous insufficiency is the initial sign of venous dermatitis. Staphylococcus aureus is found in more than $90 \%$ of stasis dermatitis.

Culture reports showed that the staphylococcus aureus and E.coli confirmed the diagnosis. The patient was prescribed with rational antibiotics as per the culture sensitivity test and regular topical application. The infected area was washed with saline and dressed regularly. The prognosis chart was maintained and monitored regularly for every 10 hours. The patient had symptomatic recovery during the course in the hospital.

The patient was counseled accordingly as regular sit-ups, with points focusing disease condition, therapy prescribed.

The patient counseling points include life style changes along with dietary restrictions.

1. Maintenance of the skin with liberal use of emollients and avoidance of aggravating factors.

2. Bathing or showering should be done with warm water not hot water.

3. Prefer cotton clothing rather than wool or any other clothes as they may cause irritation to the skin. 
4. Avoid extreme temperature which may irritate the skin $\&$ also avoid using fabric conditioners which may cause irritation to skin.

5. Rinse the clothes properly as the left over detergents in the clothes may also cause allergies or irritation.

6. The infected area should be cleaned with non-irritant soap or non-soap cleansers.

7. Shortening the fingernails helps to prevent further damage from scratching.

8. After bathing, the infected area should be part dried and application of moisturizers like liquid paraffin can be recommended.

9. Clean the hands before and after touching the infected area. Bandages should be changed every day to prevent further infections.

10. Cow's milk, eggs, soya, wheat, fish and nuts are not advisable as it may trigger the symptoms.

11. Dust and mites may cause allergies to the patient. Reducing the mites and dust may prevent further infections.

12. Increasing the adequate amount of fluid especially water helps the body to fight against cellulitis. Probiotics like yoghurt helps to recover from cellulitis.

13. Overdosing or overusing the emollients is not advisable as it does not contain active ingredients it cannot be absorbed through the skin.

14. Emollients should be used daily. Stopping the emollient when the skin appears good is a common mistake. Patches of inflammation, which has been prevented, may flare up again.

15. Stress may flare up the condition. Hence yoga or meditation can be habituated which readily helps in reducing the stress.

16. Consult the doctor before taking OTC medication.

17. Do not stop or change the dose of medication without consulting the doctor.

18. Skipping the medication is not advisable. In case of a missed dose, one can take the medication as soon as they remember. If it is time for next dose he can skip the dose for next session. ${ }^{[5-10]}$.

\section{Conclusion:-}

The patient was treated with rational antibiotic with regular counselling sessions. The patient was counselled regarding the disease including etiology and aggravating factors, treatment and lifestyle modifications. The patient was discharged after the improvement in the clinical conditions. Additionally the patient was warned not to take any over the counter medications and also herbal medications which may aggravate the present condition. The pharmacist plays an important role in analyzing the case study in order to provide excellence in clinical outcomes and to improve the patient's quality of life.

\section{References:-}

1. Jindal R, Sharma NL, Maharajan VK, Tegta GR. Contact Sensitization in Venous Eczema: Preliminary results of patch testing with Indian Standard series and topical medicaments. Indian J Dermatol Venereol Leprol 2009; 75(2): 136-141.

2. Halim K, McElroy MJ, Lewis JA. The Management of Stasis Dermatitis and Chronic Venous Insuffiency in Patients Refractory to Conservative Therapies. Harvard Medical Student Review 2015; 22(1): 1-23.

3. Shankar S.V, Shariff V.N.S.A, Nirmala S. Clinical-epidemiological study of stasis eczema. Int J Res Med Sci 2017 Sep; 5(9): 3921-3928.

4. Sabishruthi.S, Jeyamani V.P, Punitha.S, Kavitha.S, Jose F.J. Acute Eczema with Cellulitis and Eruption on Both Legs- Case Study. IJPPR Nov2017;10(4): 243-247.

5. Gray S, Anderson M, Dublin S, Hanlon J, Hubbard R, Walker R, Yu O, Crane P, Larson E. Cumulative Use of Strong Anticholinergics and Incident Dementia. JAMA Inter Med.2014; 175: 401-7.

6. Wong I.T.Y, Tsuyuski R.T, Melville AC, Doiron P, Drucker A.M, Guidelines for the management of atopic dermatitis (eczema) for Pharmacists. Can Pharm J (Ott). 2017 Sep-Oct; 150(5): 285-297.

7. Eichenfield LF, Tom WL, Berger TG, et al. Guidelines of Care for the management of atopic dermatitis: section 2. Management and treatment of atopic dermatitis with topical therapies. J Am Acad Dermatol 2014; 71(1): 116-32.

8. Sidbury R, Tom WL, Bergman JN, et al. Guidelines of care for the management of atoic dermatitis part 4: prevention of disease flares and use of adjunctive therapies and approaches. J Am Acad Dermatol 2014; 71(6): 1218-33.

9. Giam YC, Hebert AA, Dizon MV, et.al. A review on the role of moisturizers for atopic dermatitis. Asia Pac Allergy 2016; 6(2): 120-8. 
10. Godoy J.M.P.D. Treatment of stasis dermatitis using aminaphtone: a case series. Journal of Medical Case Reports 2010; 4(295): 1-3.

11. Sundaresan S, Migden MR, Silapunt S. Stasis Dermatitis: Pathophysiology, Evaluation, and Management. American Journal of Clinical Dermatology 2017; 18(3): 383-390. 